\title{
Modelling of the cylindrical geometry cooling process based on the solution of the inverse problem
}

\author{
Magda Joachimiak $^{1 *}$, and Damian Joachimiak ${ }^{1}$ \\ ${ }^{1}$ Institute of Thermal Engineering, Poznan University of Technology, 60-965 Poznan, Poland
}

\begin{abstract}
Processes of thermo-chemical treatment, such as nitriding, are used to create a surface layer of high mechanical values. When the nitriding process, often consisting of a multi-stage heating and soaking, is ended, elements being under treatment are cooled. The cooling rate depends on the massiveness and geometry of the given element. Too fast cooling can result in the formation of high temperature gradients, which leads to the element damage. This paper presents numerical analysis of a cylinder cooling. The non-linear, unsteady inverse problem for the heat equation was solved. Test examples were chosen based on experimental research conducted in the furnace for thermo-chemical treatment.
\end{abstract}

\footnotetext{
*Corresponding author: magda.joachimiak@put.poznan.pl
} 


\section{Introduction}

Machine elements are submitted to thermo-chemical treatment, such as nitriding, to create a surface layer of high mechanical properties. After the process phase consisting in a series of heating and soaking is ended, the element is cooled. It is necessary to adjust the speed of the cooling process to the massiveness and complexity of the heated element surface in such a way that the permissive thermal stresses are not exceeded. So far, research on heating the cylinder in the furnace for thermo-chemical treatment was conducted. To determine thermodynamic parameters, the inverse problem for the heat conduction equation was solved $[1,2]$. Research on the treatment of metal elements with laser are presented in papers [3,4]. To analyze the heat flow, the solution to the inverse problem was applied. Inverse problems were also used to investigate the heat flow in the aluminum solid block during the process of water cooling [5].

Computing thermal stresses can be done based on temperature distribution in the element being cooled. They can be determined by solving the direct heat conduction problem if the temperature, the heat flux or the combined radiation convection heat transfer coefficient (CHTC) on the boundary is known. Temperature measurement on the boundary of the element being treated is impossible during the processes of thermochemical treatment or is subject to a great error. However, it is possible to measure temperature inside the cooled element and to determine the boundary condition by solving the inverse problem [6]. Inverse problems are ill-conditioned numerically [7]. Many studies on methods for solving inverse problems and on their stability were conducted [8-11], with particular focus on cylindrical geometries, what was discussed in papers [12-14]. Determination of the heat transfer coefficient for the cooling process by solving the inverse problem with the use of the Trefftz method was presented in the paper [15]. Today, inverse problems are widely used to determine the temperature, the heat flux and the heat transfer coefficient in elements of machines and thermal devices during heating and cooling processes [16-22]. In this paper, numerical research on the solution to the inverse problem for cooling the cylinder is presented.

\section{Calculation model}

The process of heat flow in the cylinder is described by the heat conduction equation

$$
\rho(T) c(T) \frac{\partial T}{\partial t}=\operatorname{div}(\lambda(T) \nabla T)
$$

with the initial condition

$$
T(r, t=0)=T_{0}
$$

Temperature on the boundary of the cylinder during the time of cooling was sought

$$
T(r=R, t)=T_{b}(t)
$$

To that end, the boundary inverse problem was solved. Temperature measurement inside the cylinder was assumed. The distance between the calculated and measured values at points of temperature measurement is described by the functional of the following form

$$
I\left(T_{b}\right)=\sum_{l=1}^{L}\left(T_{c}\left(r_{l}, T_{b}\right)-T_{m}\left(r_{l}\right)\right)^{2}
$$

which was next minimized. The heat flux was calculated with the use of the Fourier's law

$$
q=-\lambda(T) \frac{\partial T}{\partial n}
$$

Obtained distribution of temperature in the cylinder and the heat flux on the cylinder boundary enable determining the value of the CHTC, noted as follows

$$
h=\frac{q}{T_{b}-T_{g}}
$$

Details of the calculation model for the heating process were given in papers $[1,13]$.

\section{Numerical tests}

For calculations, the assumed temperature of the boundary was of the following form

$$
T_{b}=550 \exp \left(-1.72 \cdot 10^{-4} t\right)
$$

Numerical tests were performed for the given function of the cooling rate set-point FC and for the gas temperature obtained from the experimental tests in the furnace for thermo-chemical treatment (Fig. 1) [1]. Based on the above functions the assumed distributions of the CHTC (Fig. 2) and of the heat flux (Fig. 3) were determined for the process of the cylinder cooling. The time of cooling was divided into three intervals, what is marked with vertical lines in figures 2 and 3 . The first time interval $\left[t_{1}, t_{2}\right]$, where $t_{1}=0 \mathrm{~s}$ and $t_{2}=1000 \mathrm{~s}$, is the interval, in which great changes of the CHTC are observed. The second interval $\left[t_{2}, t_{3}\right]\left(t_{3}=10000 \mathrm{~s}\right)$ is characterized by a significantly lower variability of the CHTC. The third interval (above $t_{3}=10000 \mathrm{~s}$ ) is the interval in which the cylinder temperature is below $100{ }^{\circ} \mathrm{C}$. Then, in many cases, the furnace is shut off and opened. Relatively small differences between the gas temperature and the cylinder boundary temperature are observed at that time. This results in the CHTC (fig. 2) and the heat flux (fig. 3) values overstating and in their fluctuations. This time interval is unimportant and it is omitted in the further analysis.

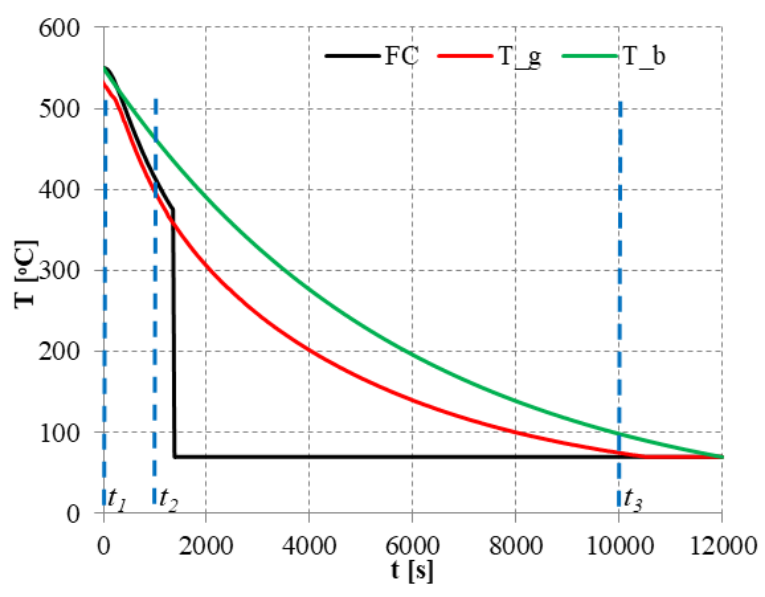


Fig. 1. Distribution of temperature of the cooling rate setpoint FC, of the gas temperature $T_{\mathrm{g}}$ and of the cylinder boundary $T_{\mathrm{b}}$ over time

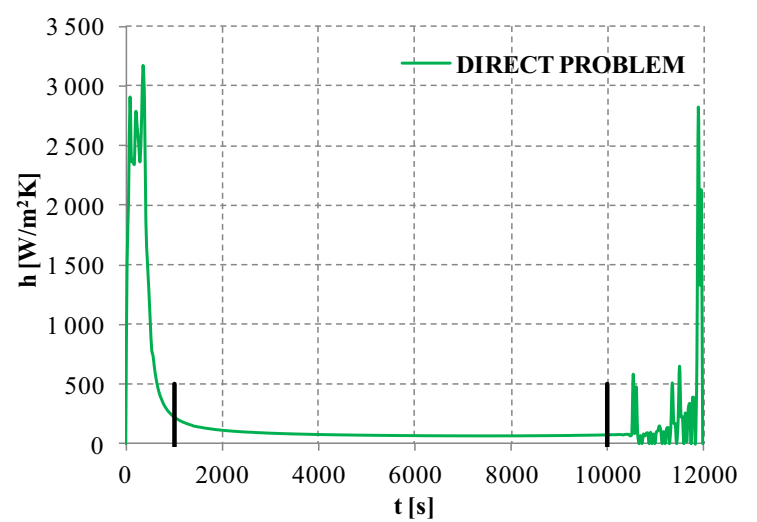

Fig. 2. The given distribution of the CHTC over time

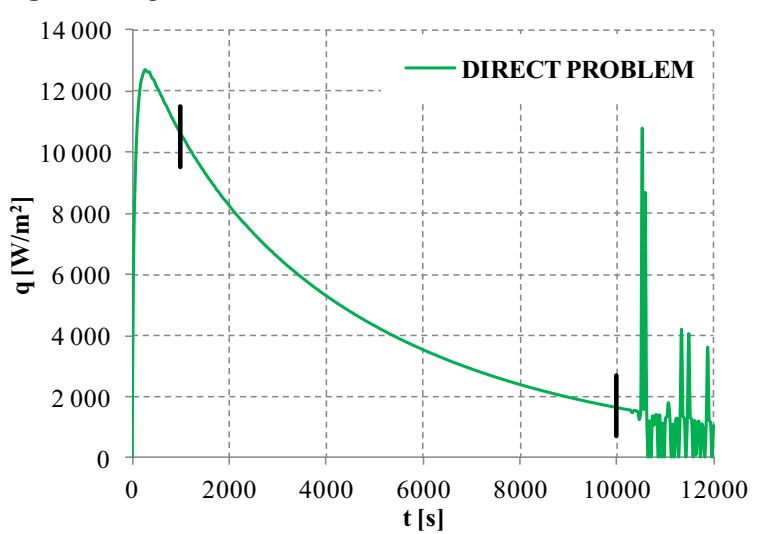

Fig. 3. The given distribution of the heat flux over time

Based on the given distributions of temperature of the cylinder boundary and of the gas temperature the direct problem was solved. Obtained temperature values inside the cylinder were next taken for numerical test as the measured values. The temperature measurement was taken on radii $r_{1}=48 \mathrm{~mm}$ and $r_{2}=46 \mathrm{~mm}$. The error in thermocouples installation was $\pm 0.2 \mathrm{~mm}$. Values of the error in temperature measurement in the function of the measured temperature are shown in figure 4. To verify the program operation, the following numerical tests were carried out:

- $\quad$ Test 1 (INVERSE PROBLEM) no disturbance to measured values

- $\quad$ Test 2 (INVERSE PROBLEM PLUS)

$$
\begin{aligned}
& \Delta T_{1}=\Delta T\left(r_{1}\right)=E T \\
& \Delta T_{2}=\Delta T\left(r_{2}\right)=E T \\
& \Delta r_{1}=-0.2 \mathrm{~mm} \\
& \Delta r_{2}=-0.2 \mathrm{~mm}
\end{aligned}
$$

- Test 3 (INVERSE PROBLEM MINUS)

$$
\begin{aligned}
& \Delta T_{1}=\Delta T\left(r_{1}\right)=-E T \\
& \Delta T_{2}=\Delta T\left(r_{2}\right)=-E T \\
& \Delta r_{1}=0.2 \mathrm{~mm} \\
& \Delta r_{2}=0.2 \mathrm{~mm}
\end{aligned}
$$

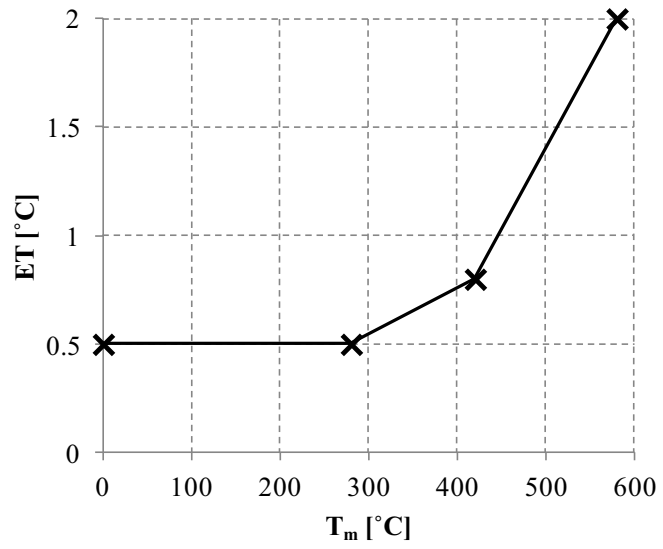

Fig. 4. Values of the error in temperature measurement in the function of the measured temperature

For all numerical tests, similar values of temperature of the cylinder boundary were obtained for the time interval $\left[t_{1}, t_{2}\right]$ (Fig. 5). The greatest differences of temperature, the CHTC and the heat flux for the time interval $\left[t_{1}, t_{2}\right]$ were obtained in test 2 (INVERSE PROBLEM PLUS), what is shown in figures from 5 to 7. Solving the inverse problem with undisturbed input data, we obtained solution being very similar to the solution to the direct problem. For tests which included the disturbance to the measuring temperature (test 2 and 3), determined values of the heat flux fluctuates around functions obtained from the direct problem. Results in the first time units differ from the assumed distribution of the heat flux. These differences decrease in next time units (Fig. 6). We obtained values of the CHTC similar to the assumed distribution for test 3 and significantly different values for test 2 (Fig. 7).

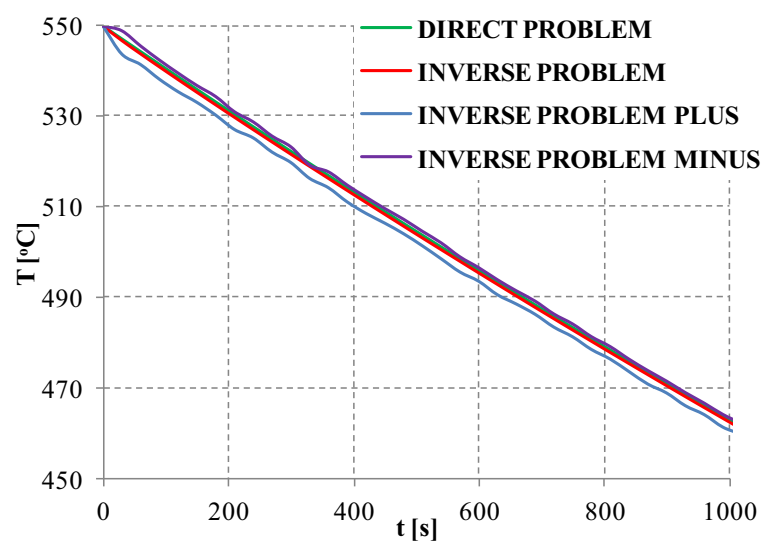

Fig. 5. Distribution of temperature for the time interval $\left[t_{1}, t_{2}\right]$

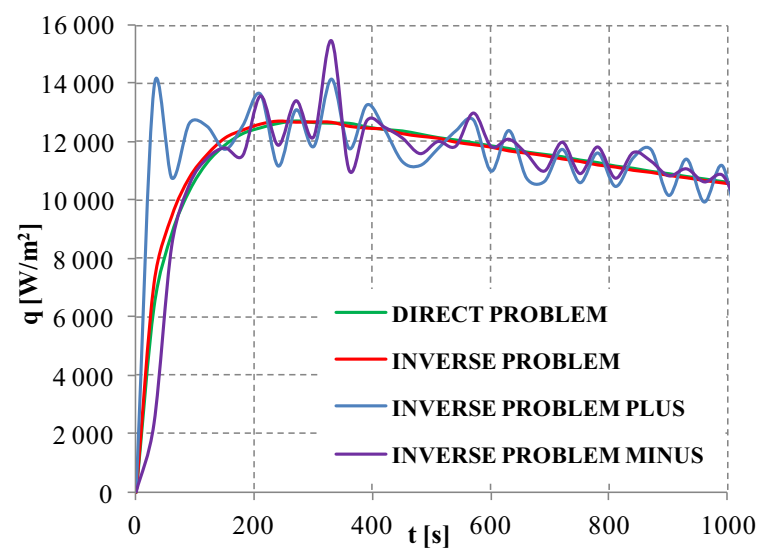


Fig. 6. Distribution of the heat flux for the time interval $\left[t_{1}, t_{2}\right]$

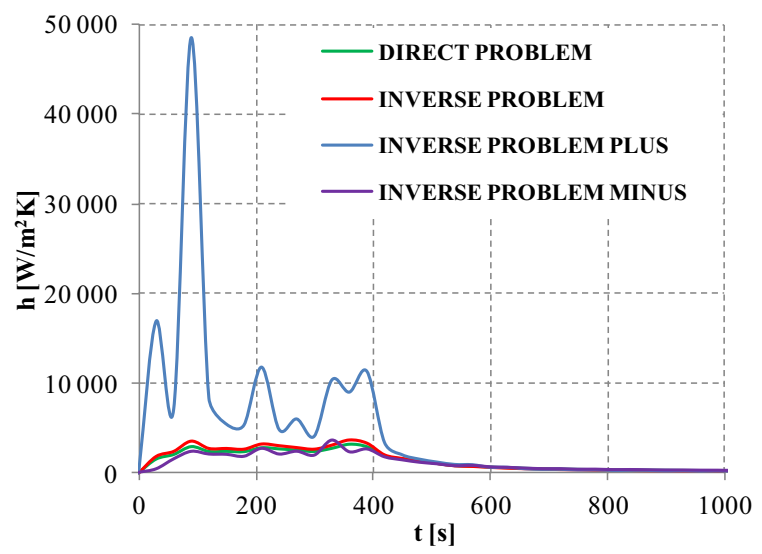

Fig. 7. Distribution of the CHTC for the time interval $\left[t_{1}, t_{2}\right]$

For the time interval $\left[t_{2}, t_{3}\right]$, values of the CHTC (Fig. 8) and of the heat flux (Fig. 9) were investigated with the maximum overstating (test 2) and the maximum understating (test 3, Fig. 10,11) of the measured values resulting from the maximum error in the measurement and from the thermocouples displacement. Despite the assumed disturbances, a stable solution to the inverse problem was obtained.

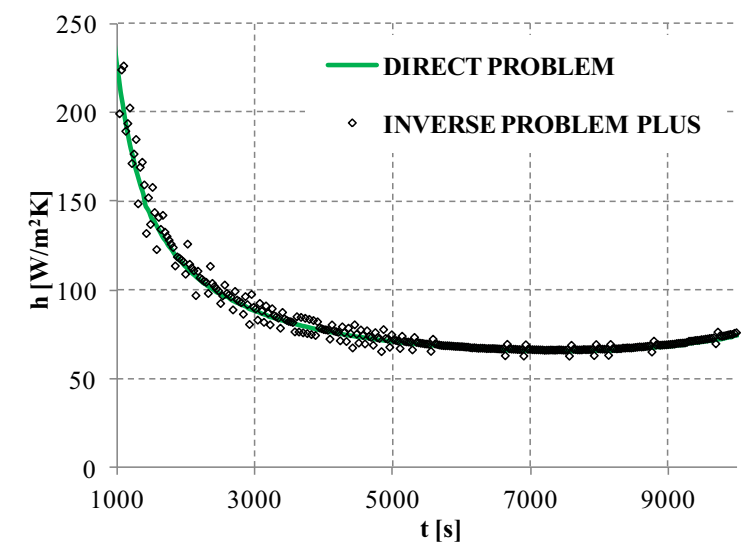

Fig. 8. Distribution of the CHTC for test 2

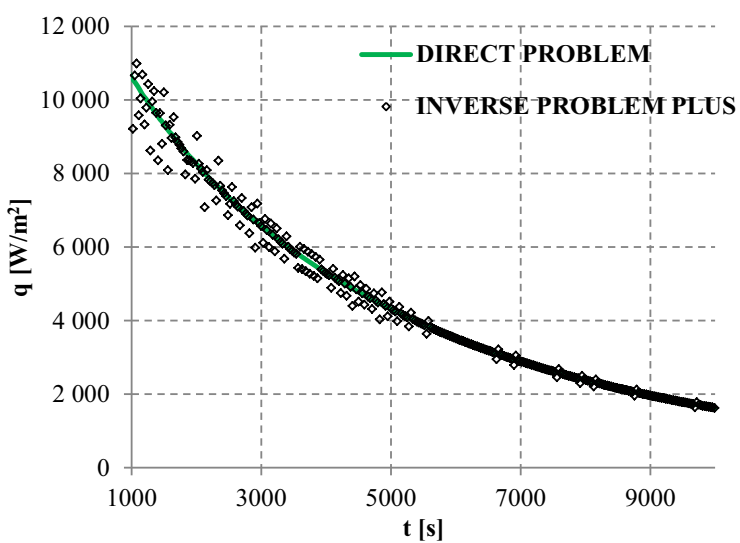

Fig. 9. Distribution of the heat flux for test 2

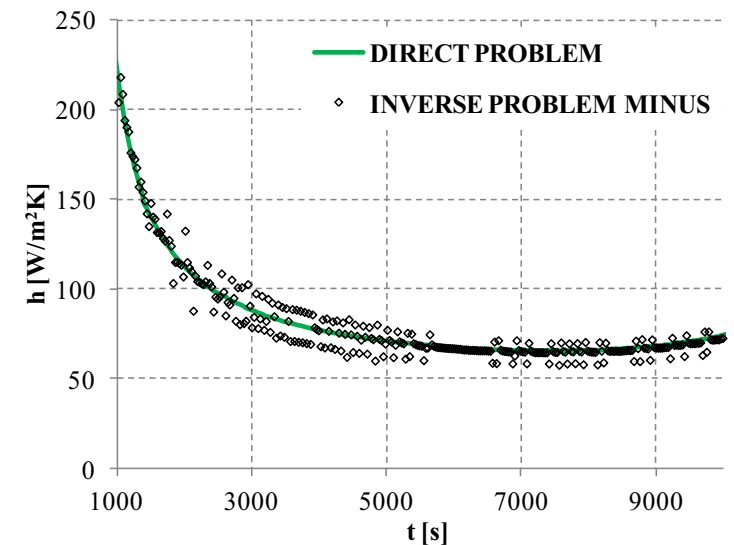

Fig. 10. Distribution of the CHTC for test 3

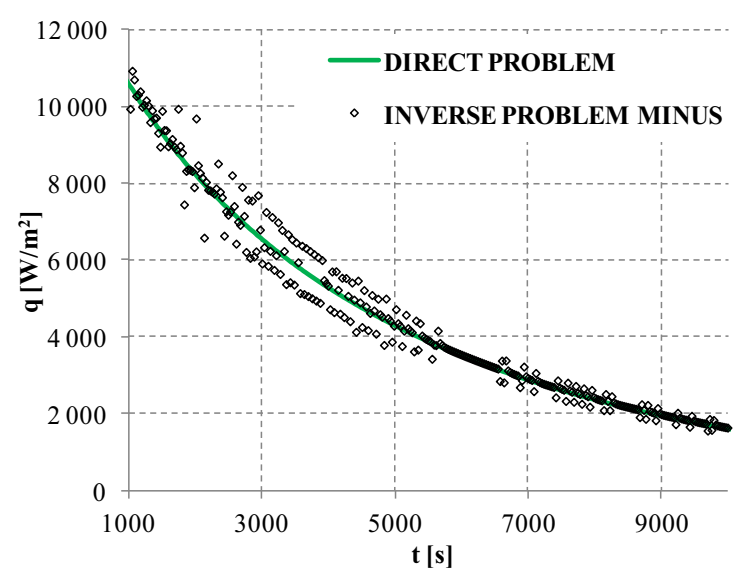

Fig. 11. Distribution of the heat flux for test 3

Differences between the solution to the direct problem and to the inverse problem were described by the maximum absolute norm

$$
N_{1}(f)=\max _{t \in\left[t_{1}, t_{2}\right]}\left|f_{D P}(t)-f_{I P}(t)\right|
$$

and by the relative norm

$$
N_{2}(f)=\frac{\max _{t \in\left[t_{1}, t_{2}\right]}\left|f_{D P}(t)-f_{I P}(t)\right|}{\max _{t \in\left[t_{1}, t_{2}\right]} f_{D P}(t)}
$$

Norms $N_{1}$ and $N_{2}$ were calculated for distributions of temperature, the CHTC and the heat flux in the time interval $\left[t_{2}, t_{3}\right]$. Results are summarized in table 1 . For the undisturbed measuring data, the solution to the inverse problem gave results being very close to those being assumed. Differences achieved up to $0.106 \%$ for the temperature, $0.36 \%$ for the heat flux and $0.81 \%$ for the CHTC, what respectively corresponded to 0.49 ${ }^{\circ} \mathrm{C}, 37.84 \mathrm{~W} / \mathrm{m}^{2}$ and $1.79 \mathrm{~W} / \mathrm{m}^{2} \mathrm{~K}$. Having regard to the overall impact of the maximum error in thermocouples installation and the maximum error in temperature measurement, these values slightly increased and achieved up to $2.37{ }^{\circ} \mathrm{C}$ (test 2), what corresponded to $0.51 \%$. For the heat flux, the greatest values of norms $N_{1}$ and $N_{2}$ were obtained for test 3 . The absolute norm $N_{1}$ for the heat flux was $1433.2 \mathrm{~W} / \mathrm{m}^{2}$, and the norm $N_{2}$ was $13.58 \%$, whereas for the CHTC these values were the greatest for test 2 and achieved $21.84 \mathrm{~W} / \mathrm{m}^{2} \mathrm{~K}$ and $9.91 \%$, respectively. The presented results prove that the solution to the inverse problem is slightly sensitive to input data disturbances. 
Table 1

\begin{tabular}{|c|c|c|c|c|}
\hline \multicolumn{2}{|c|}{} & IP & IP PLUS & IP MINUS \\
\hline \multirow{3}{*}{$N_{1}$} & $T_{b}\left[{ }^{\circ} \mathrm{C}\right]$ & 0.49 & 2.37 & 0.69 \\
\cline { 2 - 5 } & $q\left[\mathrm{~W} / \mathrm{m}^{2}\right]$ & 37.84 & 1344.25 & 1433.20 \\
\cline { 2 - 5 } & $h\left[\mathrm{~W} / \mathrm{m}^{2} \mathrm{~K}\right]$ & 1.79 & 21.84 & 20.17 \\
\hline \multirow{3}{*}{$N_{2}$} & $q[\%]$ & 0.36 & 12.74 & 13.58 \\
\cline { 2 - 5 } & $T_{b}[\%]$ & 0.106 & 0.51 & 0.15 \\
\cline { 2 - 5 } & $h[\%]$ & 0.81 & 9.91 & 9.16 \\
\hline
\end{tabular}

\section{Conclusion}

This paper presented numerical tests for a non-linear, unsteady inverse problem. Research investigated the process of cooling the cylinder. Tests were performed with respect to cooling massive elements. A stable solution to the inverse problem for the temperature with regard to maximum possible disturbances to measurement was obtained. For the case of undisturbed input data, the CHTC and the heat flux on the cylinder boundary were achieved with a great accuracy. For initial time units the solution to the inverse problem is more sensitive. Values of the CHTC and the heat flux gave satisfying results for the second time interval (from 1000 to $10000 \mathrm{~s}$ ).

\section{Nomenclature}

$f \quad$ - function

FC - function of temperature of the cooling rate setpoint

ET - error in temperature measurement, $\left[{ }^{\circ} \mathrm{C}\right]$

IP - inverse problem

Subscript:

$0 \quad-$ initial (for $t=0$ )

b - boundary

c - calculated value

DP - direct problem

g - gas

IP - inverse problem

$\mathrm{m}-$ measured value

\section{References}

1. M. Joachimiak, D. Joachimiak, M. Ciałkowski, L. Małdziński, P. Okoniewicz, and K. Ostrowska, Int. J. Therm. Sci. 145, 105985 (2019)

2. M. Joachimiak, Energies 14, 2903 (2021)

3. S.-Y. Lee, and Q.-Z. Yan, Int. J. Heat Mass Transf. 105, 401 (2017)
4. S. P. Murzin, R. Bielak, and G. Liedl, Comput. Opt. 40, 679 (2016)

5. F. Berntsson, and L. Eldén, Inverse Problems in Engineering Mechanics II, 99-106 (2000)

6. O. M. Alifanov, Inverse heat Transfer Problems (Springer 1994)

7. J. Hadamard, Sur les problèmes aux dérivéespartielles et leur signification physique (Princeton Univ. Bull. 1902)

8. M. Ciałkowski, A. Olejnik, A. Frąckowiak, N. Lewandowska, and J. Mosiężny, Energy 212, $118751(2020)$

9. M. Ciałkowski, A. Olejnik, M. Joachimiak, K. Grysa, A. Frąckowiak, Int. J. Numer. Methods Heat Fluid Flow ahead-of-print (2021)

10. M. Joachimiak, Int. J. Numer. Methods Heat Fluid Flow 30, 4475 (2020)

11. M. Joachimiak, M. Ciałkowski, and A. Frąckowiak, Int. J. Numer. Methods Heat Fluid Flow 30, 1441 (2020)

12. M. Jaremkiewicz, and J. Taler, Energies, 13, 704 (2020)

13. M. Joachimiak, and M. Ciałkowski, Arch. Thermodyn. 38, 81 (2017)

14. M. Joachimiak, and M. Ciałkowski, Arch. Thermodyn. 39, 25 (2018)

15. K. Grysa, K., A. Maciag, A. Cebo-Rudnicka, and M. Walaszczyk, Eng. Anal. Bound. Elem. 95, 33 (2018)

16. A. Frąckowiak, D. Spura, U. Gampe, and M. Ciałkowski, Int. J. Numer. Methods Heat Fluid Flow 30, 1725 (2019)

17. D. Joachimiak and A. Frąckowiak, Energies 13, 5794 (2020)

18. W. Judt, Energies 13, 6141 (2020)

19. W. Judt, B. Ciupek, and R. Urbaniak, Energy 196, 117093 (2020)

20. B. Maciejewska, K. Strak, and M. Piasecka, Int. J. Numer. Methods Heat Fluid Flow 28, 206 (2018)

21. J. Taler, P. Dzierwa, M. Jaremkiewicz, D. Taler, K. Kaczmarski, M. Trojan, and T. Sobota, Energy 175, 645 (2019)

22. J. Taler, P. Dzierwa, M. Jaremkiewicz, D. Taler, K. Kaczmarski, M. Trojan, B. Węglowski, and T. Sobota, J. Therm. Stress. 42, 698 (2019) 